\title{
BRAF mutation in cytologically indeterminate thyroid nodules: after reclassification of a variant thyroid carcinoma
}

This article was published in the following Dove Medical Press journal:

OncoTargets and Therapy

\author{
Warut Pongsapich' \\ Cheerasook \\ Chongkolwatana' \\ Naravat Poungvarin ${ }^{2}$ \\ Kanchana Amornpichetkul ${ }^{3}$ \\ Nutthaya Piyawattayakorn' \\ Pichpisith Vejvisithsakul ${ }^{2}$ \\ Prachya \\ Maneeprasopchoke'
}

'Department of Otorhinolaryngology, Faculty of Medicine, Siriraj Hospital, Mahidol University, Bangkok 10700 ,

Thailand; ${ }^{2}$ Department of Clinical

Pathology, Faculty of Medicine, Siriraj Hospital, Mahidol University, Bangkok 10700, Thailand; ${ }^{3}$ Department of

Pathology, Faculty of Medicine, Siriraj Hospital, Mahidol University, Bangkok 10700, Thailand
Correspondence: Prachya

Maneeprasopchoke

Department of Otorhinolaryngology, Faculty of Medicine Siriraj Hospital, Mahidol University, 2 Wanglang Road, Bangkoknoi, Bangkok 10700, Thailand

Tel +6624198040

Fax +66 24198044

Email prachya.man@mahidol.ac.th
Purpose: Fine-needle aspiration biopsy (FNAB) is regarded by the Bethesda system as the gold-standard investigation for stratifying the risk of malignancy of a thyroid nodule. However, some limitations affect the adequacy of the obtained materials, resulting in $30 \%$ of the cytological results remaining in the indeterminate category. We aimed to investigate the diagnostic value of the BRAF mutation in cytologically indeterminate thyroid nodules after the reclassification of a variant thyroid carcinoma.

Patients and methods: In this prospective diagnostic study, 76 patients with FNAB findings of atypia of undetermined significance (AUS) and suspicious for malignancy (SUS) were included. The BRAF V600 mutation from FNAB was confirmed by a PCR-based method (Sanger sequencing combined with allele-specific real-time PCR techniques) and immunohistochemistry (IHC). Pathological specimens and features, including noninvasive follicular thyroid neoplasm with papillary-like nuclear features (NIFTP), were reviewed and compared to the FNAB results. Results: Using the PCR-based method, the BRAF mutation was positive in 13/76 cases (17.1\%), with the diagnostic values of $16.7 \%$ sensitivity, $100 \%$ specificity, $100 \%$ positive predictive value (PPV), and $82.8 \%$ negative predictive value (NPV) in the AUS compared to $73.3 \%$ sensitivity, $100 \%$ specificity, $100 \%$ PPV, and 20\% NPV in the SUS. For the IHC technique, only 20 of the 76 cytological specimens were qualified for testing. The BRAF mutation was positive in $13 / 20$ cases, with the diagnostic values of $100 \%$ sensitivity, $63.6 \%$ specificity, $42.9 \%$ PPV, and $100 \%$ NPV in the AUS compared to $100 \%$ sensitivity and PPV in the SUS. The BRAF mutation was not found in the pathological reports for NIFTP.

Conclusion: The malignancy rate is high in our data, with specific and acceptable accuracy rates for the BRAF mutation from FNAB found by using the PCR-based method. NIFTP has been introduced after the pathological reclassification. Molecular diagnosis might be useful to establish the nature of the disease.

Keywords: papillary thyroid carcinoma, fine-needle aspiration, BRAF mutation, indeterminate thyroid nodules, noninvasive follicular thyroid neoplasm with papillary-like nuclear features, NIFTP, Thailand

\section{Introduction}

Thyroid cancer is found in $5 \%-10 \%$ of the thyroid nodules, ${ }^{1}$ with papillary thyroid carcinoma (PTC) being the most common type of thyroid malignancy. ${ }^{2}$ Fine-needle aspiration biopsy (FNAB) is widely accepted as a tool for cancer risk stratification. In the Bethesda reporting system, $\sim 30 \%$ of the cytologically indeterminate results are reported with diagnostic and therapeutic difficulties. ${ }^{3}$ The indeterminate category designated as PTC includes atypia of undetermined significance (AUS) and suspicious 
for malignancy (SUS). ${ }^{3}$ According to the 2015 American Thyroid Association (ATA) guidelines, ${ }^{4}$ molecular testing could be suggested as an option for diagnosis and management planning (rating $\mathrm{C}$ ). Following a malignancy rate of $5 \%-15 \%$ in the AUS group, it is thus recommended to repeat the FNAB or perform molecular testing. In cases of inconclusive results from molecular testing, a surgical diagnostic lobectomy can be applied. By comparison, due to the higher rate of malignancy $(60 \%-75 \%)$ in the SUS group, surgery is required for definite diagnosis and treatment. Nonetheless, molecular testing may be considered as a clinical adjunctive tool that, combined with ultrasound, aids in the making of decisions on the extent of the surgery. ${ }^{5}$

In recent decades, there have been many studies on gene mutations such as BRAF, RAS, RET/PTC, and PAX8/ PPAR $\gamma$. These mutations have been proposed as molecular markers for thyroid cancer, ${ }^{6}$ with the BRAF V600E mutation being the most common genetic event in $\sim 45 \%$ of the PTCs. ${ }^{7}$ A mutation at V600E of the BRAF gene, involving substitution of glutamate (E) for valine (V) at codon 600 , activates the serine/threonine kinase RAF type B and the whole signaling pathway of MAPK (MAPK kinase), resulting in the development of thyroid cancer. ${ }^{8}$

From the study by Xing et al, ${ }^{9}$ BRAF V600E was associated with a poorer prognosis and more aggressive tumor behaviors (extrathyroidal extension and lymph node metastasis). Furthermore, BRAF V600E is also related to a higher rate of recurrence and mortality. ${ }^{7,10}$ Nevertheless, there have been various methods for detecting the BRAF mutation from FNAB samples. Sanger sequencing and allele-specific PCR techniques are used to identify the mutation from freshly aspirated samples, ${ }^{11}$ whereas immunohistochemistry (IHC) staining is used on the formalin-fixed cell block left in the needle. ${ }^{12}$

The term "noninvasive follicular thyroid neoplasm with papillary-like nuclear features" (NIFTP) has recently been reclassified separately from differentiated thyroid cancer. ${ }^{13}$ Formerly, NIFTP was classified as a type of encapsulated follicular variant of PTC. Due to its indolent nature, no further management is suggested after diagnostic hemithyroidectomy. NIFTP is neither definitely benign nor definitely malignant. ${ }^{14}$ The risk of malignancy for each of the indeterminate categories of the Bethesda reporting system needs to be adjusted after the reclassification of NIFTP, and NIFTP will be calculated as both a malignant and a benign tumor in accordance with Bethesda (2017). ${ }^{15}$

In this study, we aimed to determine the diagnostic value of the BRAF mutation in patients with Bethesda cytologically indeterminate thyroid nodules from FNAB after reclassification.

\section{Objectives}

The primary objective was to determine the diagnostic value of the BRAF mutation in patients with Bethesda cytologically indeterminate thyroid nodules from FNAB. The secondary objective was to compare the efficacy of a PCR-based technique and IHC for the detection of the BRAF mutation.

\section{Materials and methods \\ Study design}

This prospective diagnostic study of patients with cytologically indeterminate thyroid nodules was conducted at the Department of Otorhinolaryngology, Faculty of Medicine, Siriraj Hospital. The repository of biological products from cell cytology was used for the in vitro, laboratory-based study. This study was approved by the Institutional Review Board, Faculty of Medicine, Siriraj Hospital, Mahidol University (no. 740/2556, EC2) and conducted in accordance with the Declaration of Helsinki.

\section{Study population}

The study was conducted from July 2014 to October 2016. Adult, Thai patients with preoperative thyroid FNAB reports of AUS, as shown in Figure 1, or SUS, as shown in Figures 2 and 3, according to the Bethesda cytological reporting system were included. ${ }^{3}$ A thyroidectomy was done in all patients, based on the FNAB, ultrasound, and clinical presentations.

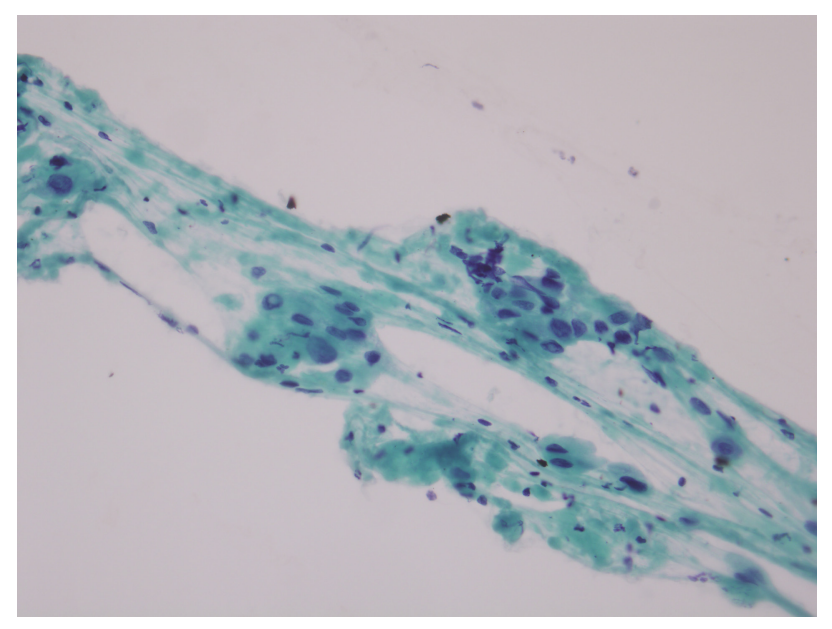

Figure I Atypia of undetermined significance: nuclear enlargement and molding are noted in small follicular cell clusters from paucicellular smears of thyroid fine-needle aspiration biopsy specimen.

Note: Some possible intranuclear pseudoinclusions are observed (Papanicolaou/ $400 \times)$. 


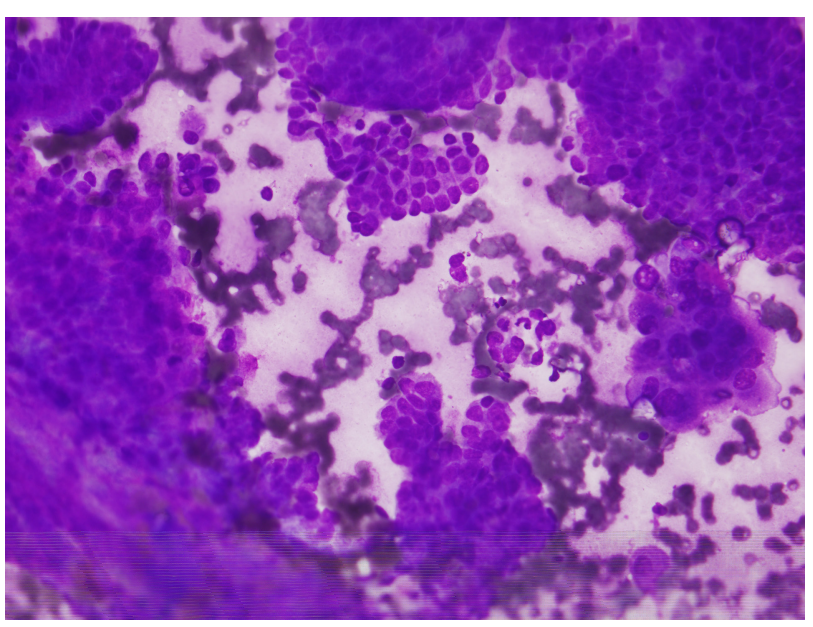

Figure 2 Suspicious for malignancy: few fragments of three-dimensional papillary structure are shown.

Note: Enlarged nuclei of follicular cells are observed (Diff Quik/400x).

All patients gave written informed consent after receiving complete information on treatment options and making decision with their surgeons. Uncertain nodules $<1 \mathrm{~cm}$ in diameter were excluded.

\section{Intervention}

FNAB

FNAB was performed intraoperatively at the suspected thyroid nodule as the preoperative FNAB. The cytology samples were collected by using a 25 -gauge needle and subsequently diluted with $0.9 \%$ normal saline to $3 \mathrm{~mL}$ before being sent for BRAF V600 mutation detection using the PCR-based technique. The leftover cells in the syringe were collected for cell block processing and sent for IHC.

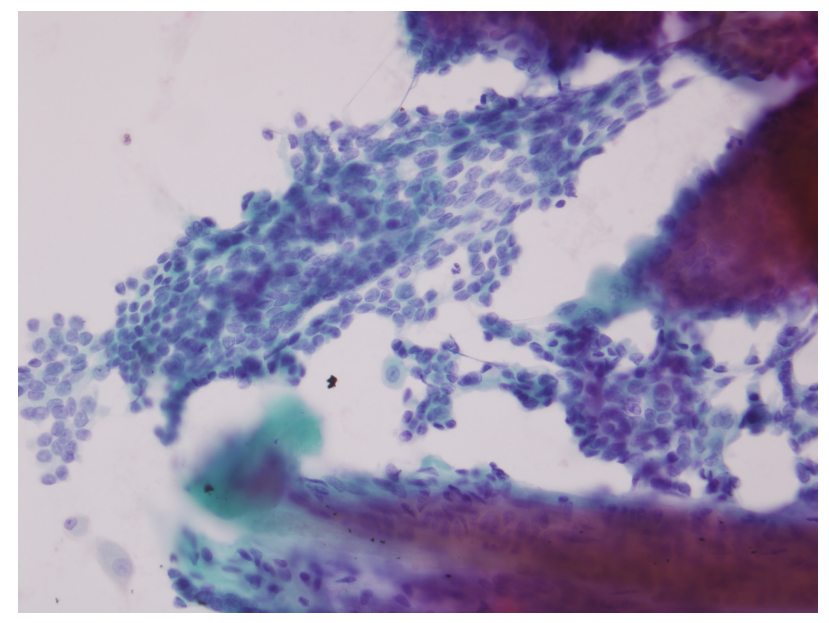

Figure 3 Suspicious for malignancy: another spot from the same case.

Note: The characteristic powdery nuclear chromatin and nuclear grooves are observed (Papanicolaou/400×).

\section{Analysis of BRAFV600 mutation}

The PCR-based method of Sanger-sequencing technique combined with allele-specific real-time PCR was used. We isolated the genomic DNA from the clinical specimens using the cobas DNA Sample Preparation Kit (Hoffman-La Roche Ltd., Basel, Switzerland). The 152-bp DNA fragment of $B R A F$ exon 15 (NM_004333.5) was amplified by PCR. The oligos used for sequencing were BRAF51 5'-TGTAAAAC GACGGCCAGTCCTTTACTTACTACACCTCAG-3' and BRAF31 5'-CAGGAAACAGCTATGACCTAGCCT CAATTCTTACCATCC-3' with minor modification from the study by Yanus et al. ${ }^{16}$ The underlined sequences were the M13 universal primer sequences. We performed bidirectional Sanger sequencing on an ABI 310-automated DNA sequencer (Thermo Fisher Scientific, Waltham, MA, USA). Laboratory-developed allele-specific real-time PCR was used to confirm the BRAF V600 mutation in all cases. We used the reference standard DNA (Horizon Discovery, Cambridge, UK) containing $0 \%$ or $1 \%$ BRAF V600E mutant allele as negative and positive controls, respectively. This assay could detect the BRAF V600 mutation presenting in at least $1 \%$ within the background of wild-type DNA. ${ }^{17}$

\section{IHC}

The immunohistochemical method using anti-BRAF V600E antibody (catalog no. 790-4855; Ventana Medical Systems, Tucson, AZ, USA) ${ }^{12}$ was used. Cytological specimens from FNAB were prepared into cell blocks for the BRAF mutation analysis. Positive cytoplasmic staining was demonstrated in a BRAF mutation, as shown in Figure 4, while the absence of cytoplasmic staining led to the diagnosis of a negative BRAF mutation.

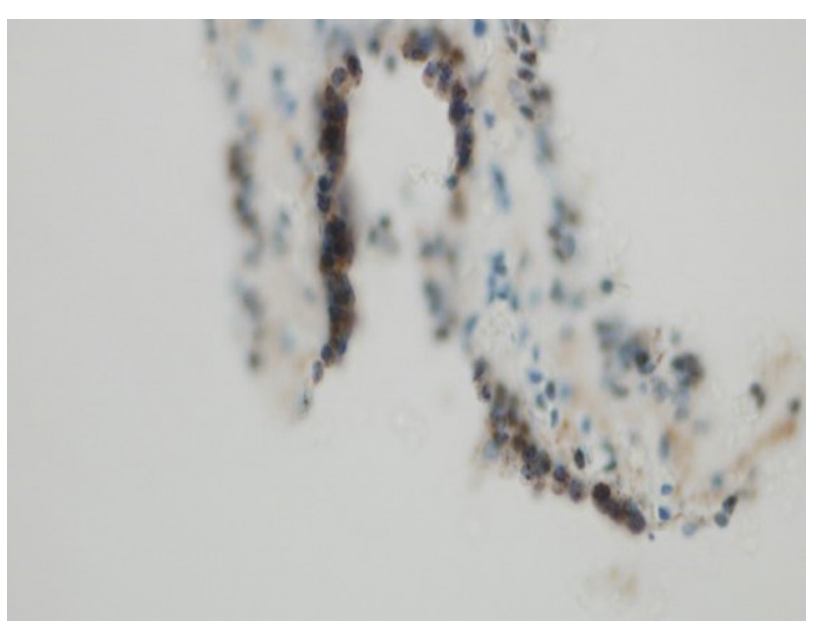

Figure 4 Positive BRAF mutation: positive cytoplasmic staining of BRAF mutation (immunohistochemistry/100X). 


\section{Pathological review}

After each operation, the entire thyroid specimens were sent to the Department of Pathology, Faculty of Medicine, Siriraj Hospital. The pathological specimens were reviewed and compared with the FNAB results, which were blinded to each other. After reclassification of the variant thyroid carcinoma in 2016, the pathological specimens were reevaluated by using the criteria of "noninvasive follicular thyroid neoplasm with papillary-like nuclear features"13 by a single, experienced cytopathologist.

\section{Statistical analyses}

Demographic data were presented using descriptive statistics. The two-sample $t$-test and Mann-Whitney test were used to compare the quantitative variables of the two groups. Fisher's exact test was employed to compare the qualitative variables of both groups. The histopathology report was the gold standard for calculations. The diagnostic values (ie, sensitivity, specificity, positive predictive value [PPV], negative predictive value [NPV], and accuracy) and 95\% CI were calculated. A $P$-value of $<0.05$ was considered to be statistically significant. Statistical analyses were performed by using the PASW 18 and MEDCALC programs.

\section{Results}

\section{Demographic data and patients' characteristics}

Of the 84 patients with indeterminate thyroid nodules from FNAB who were recruited between July 2014 and October 2016, eight were excluded because of accidentally found microcarcinoma. The remaining 76 patients $(15$ males and 61 females) were included in the analysis. The mean age of those patients was 52.1 \pm 15.3 years (minimum: 22 years, maximum: 86 years). The baseline demographic data, patients' characteristics, and cytological and pathological results are shown in Table 1.

\section{Malignancy rate}

Following the 2017 Bethesda reporting system, NIFTP had implications for the risk of malignancy.$^{15}$ The malignancy rate of the indeterminate group was $32.9 \%$ (NIFTP $\neq$ malignancy) and $35.5 \%$ (NIFTP = malignancy). The malignancy rate of the AUS was $16.7 \%$ (NIFTP $\neq$ malignancy) and $20 \%$ (NIFTP $=$ malignancy). The malignancy rate of the SUS was $93.8 \%$ for both categories.

\section{BRAF mutation rate}

Among the 76 samples of bidirectional sequencing, we could distinctly detect the BRAF mutation in eight samples,
Table I Demographic data and characteristics of 76 patients with indeterminate thyroid nodules

\begin{tabular}{l|l}
\hline Characteristics & Number (\%) \\
\hline Age (years): mean \pm SD & $52.1 \pm 15.3$ \\
$<45$ & $24(31.6)$ \\
$\geq 45$ & $52(68.4)$ \\
Sex & $15(19.7)$ \\
$\quad$ Male & $61(80.3)$ \\
Female & \\
Side & $46(60.5)$ \\
Right & $22(28.9)$ \\
Left & $8(10.5)$ \\
Isthmus & \\
Cytology & $60(73.3)$ \\
AUS & $16(26.7)$ \\
SUS & \\
Histopathology & $49(64.5)$ \\
Benign & $2(2.6)$ \\
NIFTP & $24(31.6)$ \\
Papillary thyroid carcinoma & $1(1.3)$ \\
Follicular carcinoma & \\
\hline
\end{tabular}

Abbreviations: AUS, atypia of undetermined significance; NIFTP, noninvasive follicular thyroid neoplasm with papillary-like nuclear features; SUS, suspicious for malignancy.

and allele-specific PCR was detected in five additional samples. Totally, there were 13 of the 76 cases (17.1\%) with a BRAF mutation detected from FNAB. Of those 13 cases, 2 were in the AUS and 11 were in the SUS categories. The BRAF mutation results were later compared with the histopathology, which was the gold standard for the PTC diagnosis.

\section{Diagnostic value of BRAF mutation}

In this study, we classified the neoplastic variant in the malignant group by using the PCR-based technique and IHC as described subsequently. The diagnostic values for each category of cytology using the PCR-based technique are shown in Table 2. By using the IHC technique, only 20 specimens were qualified for testing; their diagnostic values are shown in Table 3.

\section{Association of BRAF mutation status with histopathology and clinical outcomes}

Twenty-five patients of differentiated thyroid carcinoma (DTC), including 24 with PTC and one with follicular carcinoma, were analyzed for the association of the BRAF mutation with the histopathology and clinical outcomes. The mean follow-up time was $32.7 \pm 12$ months (minimum: 14 months, maximum: 47 months). The results are shown in Table 4.

\section{NIFTP}

After the review of the pathological specimens, two cases of NIFTP were diagnosed by using the consensus diagnostic 
Table 2 Diagnostic value of BRAF mutation in 76 cytologically indeterminate thyroid nodules by using the PCR-based technique (Sanger sequencing and allele-specific real-time PCR technique)

\begin{tabular}{|c|c|c|c|c|c|c|}
\hline \multirow[t]{2}{*}{$\overline{\text { Diagnostic category }}$} & \multirow[t]{2}{*}{$\mathbf{n}$} & \multicolumn{5}{|c|}{$\%$ point estimate $(95 \% \mathrm{Cl})$} \\
\hline & & Sensitivity & Specificity & PPV & NPV & Accuracy \\
\hline Indeterminate & 76 & $48.2(28.7-68.1)$ & $100(92.8-100)$ & 100 & $77.8(70.9-83.4)$ & $81.6(71.0-89.6)$ \\
\hline AUS & 60 & $16.7(2.1-48.4)$ & $100(92.6-100)$ & 100 & $82.8(78.8-86.1)$ & $83.3(71.5-91.7)$ \\
\hline SUS & 16 & $73.3(44.9-92.2)$ & $100(2.5-100)$ & 100 & $20.0(9.8-36.7)$ & $75(47.6-92.7)$ \\
\hline
\end{tabular}

Abbreviations: AUS, atypia of undetermined significance; NPV, negative predictive value; PPV, positive predictive value; SUS, suspicious for malignancy.

criteria, as shown in Figures 5 and $6 .{ }^{13}$ However, no BRAF mutations could be identified. These patients were treated as papillary microcarcinoma.

\section{Discussion}

Over the last decade, thyroid cancer has been rapidly increasing worldwide. Approximately $85 \%$ of the histologic type of thyroid cancer are PTCs. ${ }^{18,19}$ FNAB is regarded as the goldstandard investigation for stratifying the risk of malignancy of a thyroid nodule. ${ }^{15}$ However, there are some limitations that affect the adequacy of obtained materials, such as the size and location of the nodules, the quantity and quality of the specimens, technical skills, and the overlapping cytological morphology. ${ }^{20}$ As a result, $15 \%-30 \%$ of the cytological results remain in the indeterminate category. ${ }^{4}$

Meanwhile, molecular testing has been developed to overcome the limitations of FNAB with the purpose of avoiding unnecessary surgery and surgical complications in patients with indeterminate FNAB results..$^{21}$ Cohen et al ${ }^{22}$ reported an association of BRAF mutations with PTC in 2003. Thereafter, much clinical research on the BRAF mutation has been undertaken. The BRAF V600E mutation is the most common genetic event in thyroid cancer, with a high specificity for PTC. Abnormal activation of the MAPK pathway by the BRAF mutation results in carcinogenesis and the progression of cancer. ${ }^{23}$ The prevalence of the BRAF mutation in PTC has varied from $30 \%-50 \%$ in western countries ${ }^{24}$ to $80 \%$ in Korea. ${ }^{25}$ In the Thai population, Khemkha et al reported a BRAF mutation prevalence of $56 \%$ in PTC..$^{26}$ The different prevalence rates may stem from geographic factors, ethnicity, and iodine intake. ${ }^{27,28}$
In a 2016 meta-analysis by $\mathrm{Su}$ et $\mathrm{al}^{20}$ of the use of the BRAF mutation as a molecular marker for the preoperative diagnosis of thyroid malignancy in the cytologically indeterminate group, 88 studies were included. The BRAF mutation rate was $23 \%$ for the indeterminate group, with a high specificity of $99 \%$ but a relatively low sensitivity of $44 \%$, in the overall BRAF V600E analysis. In a 2017 meta-analysis by Jinih et al, ${ }^{29} 32$ studies involving 3,150 indeterminate nodules were included. The $100 \%$ specificity and $40 \%$ sensitivity found were comparable to the results of the previous analysis. The study by Jinih et al also had a subgroup analysis to detect the BRAF mutation that employed the same methods as our study. According to their results, the Sanger sequencing and allele-specific real-time PCR techniques yielded a $100 \%$ specificity, with a $45 \%$ and $32 \%$ sensitivity for each PCR-based method, respectively. Nonetheless, the IHC method resulted in a much lower specificity rate and a much higher sensitivity rate of $91 \%$ and $56 \%$, respectively.

In the present study, 76 indeterminate thyroid nodules with FNAB were prospectively studied. We included patients in both the AUS and the SUS groups due to the significant relationship with PTC. We excluded cases of follicular neoplasm or suspicious for follicular neoplasm (FN/SFN), which were mainly composed of follicular variant of papillary thyroid cancer, follicular thyroid cancer, adenomatoid hyperplasia, and follicular adenoma. All the excluded cases have a negative, or a low, prevalence of BRAF mutations and low false-negative results.

The diagnostic value of the BRAF mutation in the cytologically indeterminate samples found in this present study by using the PCR-based technique was similar to that of

Table 3 Diagnostic value of BRAF mutation in cytologically indeterminate thyroid nodules by using immunohistochemistry technique (only 20 adequate specimens from 76 patients)

\begin{tabular}{|c|c|c|c|c|c|c|}
\hline \multirow[t]{2}{*}{ Diagnostic category } & \multirow[t]{2}{*}{$\mathbf{n}$} & \multicolumn{5}{|c|}{$\%$ point estimate $(95 \% \mathrm{CI})$} \\
\hline & & Sensitivity & Specificity & PPV & NPV & Accuracy \\
\hline Indeterminate & 20 & $100(66.3-100)$ & $63.6(30.8-89.1)$ & $69.2(50.7-83.1)$ & 100 & $80(56.3-94.3)$ \\
\hline AUS & 14 & $100(29.2-100)$ & $63.6(30.8-89.1)$ & $42.9(25.6-62.1)$ & 100 & $71.4(4|.9-9| .6)$ \\
\hline SUS & 6 & $100(54.1-100)$ & & 100 & & $100(54.1-100)$ \\
\hline
\end{tabular}

Abbreviations: AUS, atypia of undetermined significance; NPV, negative predictive value; PPV, positive predictive value; SUS, suspicious for malignancy. 
Table 4 Association of BRAF mutation status on histopathology and clinical outcomes of differentiated thyroid cancer

\begin{tabular}{|c|c|c|c|}
\hline & \multicolumn{2}{|c|}{ Number (\%) } & \multirow[t]{2}{*}{$P$-value } \\
\hline & $\begin{array}{l}\text { BRAF } \\
\text { mutation } \\
\text { negative } \\
(n=\mid 2)\end{array}$ & \begin{tabular}{|l|} 
BRAF \\
mutation \\
positive \\
$(n=13)$
\end{tabular} & \\
\hline Age (years): mean $\pm S D$ & $48.3 \pm 12.7$ & $42.1 \pm 18.7$ & 0.270 \\
\hline Sex & & & 1.000 \\
\hline Male & $4(50.0)$ & $4(50.0)$ & \\
\hline Female & $8(47.1)$ & $9(52.9)$ & \\
\hline Tumor size $(\mathrm{mm})$ : mean $\pm \mathrm{SD}$ & $27.7 \pm 16.8$ & $21.3 \pm 8.4$ & 0.503 \\
\hline Cytology, Bethesda system & & & 0.015 \\
\hline AUS & $8(80.0)$ & $2(20.0)$ & \\
\hline SUS & $4(26.7)$ & II (73.3) & \\
\hline Histopathology & & & 0.480 \\
\hline Papillary thyroid carcinoma & II (45.8) & $13(54.2)$ & \\
\hline Follicular carcinoma & I & 0 & \\
\hline AJCC staging & & & 0.147 \\
\hline I & $6(37.5)$ & $10(62.5)$ & \\
\hline ॥ & I (50.0) & I (50.0) & \\
\hline III & I (33.3) & $2(66.7)$ & \\
\hline IV & $4(100)$ & 0 & \\
\hline Extracapsular invasion & & & 0.115 \\
\hline No & $4(30.8)$ & $9(69.2)$ & \\
\hline Yes & $8(66.7)$ & $4(33.3)$ & \\
\hline Lymph node metastasis & & & 1.000 \\
\hline No & $8(47.1)$ & $9(52.9)$ & \\
\hline Yes & $4(50.0)$ & $4(50.0)$ & \\
\hline Distant metastasis & & & 0.480 \\
\hline No & II (45.8) & $13(54.2)$ & \\
\hline Yes & 1 & 0 & \\
\hline Recurrence & & & 0.220 \\
\hline No & $10(43.5)$ & $13(56.5)$ & \\
\hline Yes & 2 & 0 & \\
\hline
\end{tabular}

Abbreviations: AJCC, American Joint Committee on Cancer; AUS, atypia of undetermined significance; SUS, suspicious for malignancy.

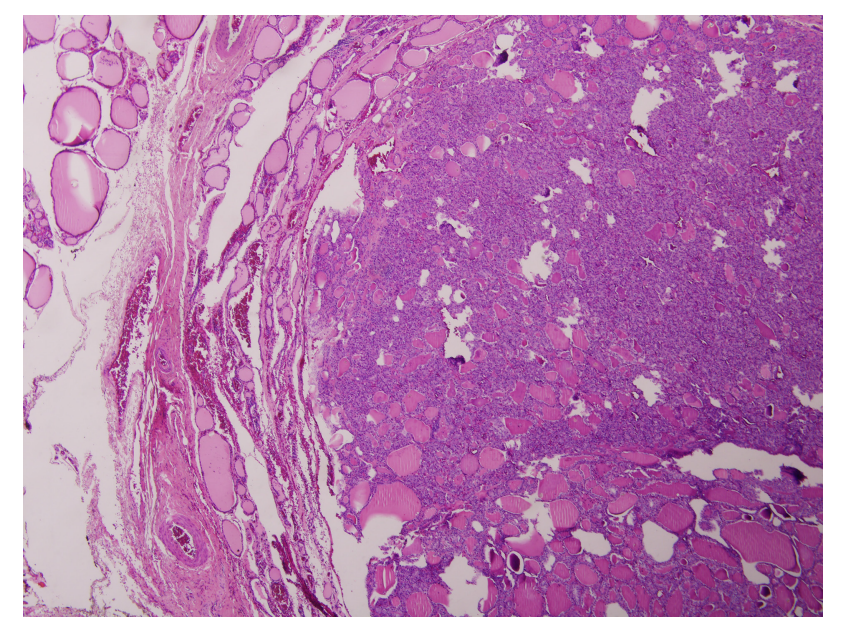

Figure 5 Noninvasive follicular thyroid neoplasm with papillary-like nuclear features: the nodule consists of mainly normo- and microfollicles without papillary growth or other architectural pattern.

Note: The circumscribed, noninvasive border is observed $(\mathrm{HE} / 40 \times)$.

Abbreviation: HE, Hematoxylin and eosin stain.

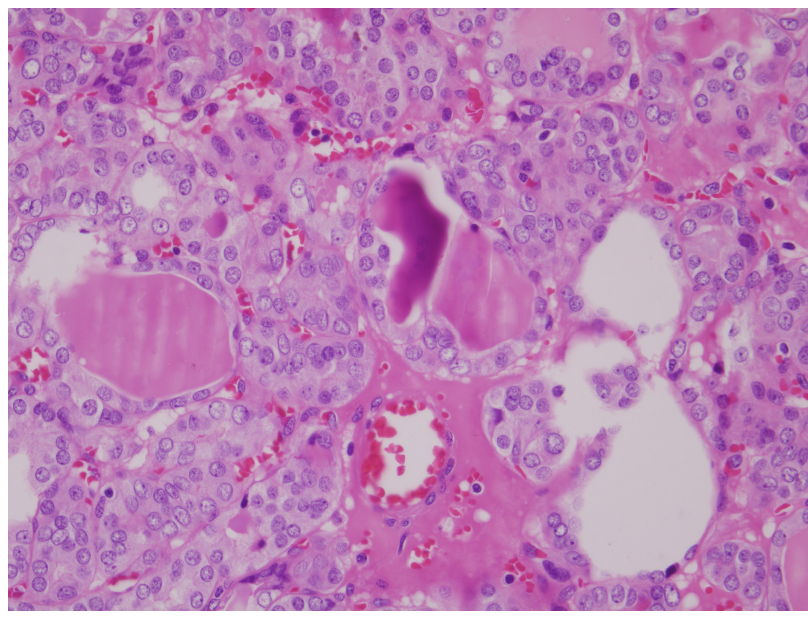

Figure 6 Noninvasive follicular thyroid neoplasm with papillary-like nuclear features: the follicular cells are enlarged and oval in shape.

Notes: Chromatin clearing is discernible. Occasional longitudinal nuclear grooves are present $(\mathrm{HE} / 400 \times)$.

Abbreviation: $\mathrm{HE}$, Hematoxylin and eosin stain.

previous studies, having high specificity and low sensitivity, as shown in Table 2. The high specificity and high PPV, as well as the acceptability of the testing accuracy, especially in the SUS group, indicate that the BRAF mutation might be useful not only to confirm a diagnosis of malignancy but also to decide the extent of the operation. In the AUS group, despite the high specificity and PPV, the low sensitivity made the BRAF mutation of limited value for clinical use. The application of a positive mutation for this test was utilized as a rule in test for malignancy, but negative mutation cases still remained in the indeterminate category.

Other genetic mutations being explored for thyroid cancer include RET-PTC, PAX8-PPAR $\gamma$ rearrangements, and RAS point mutations. ${ }^{30}$ Several panel tests for these common genes, such as the BRAF V600E mutation, have been developed for clinical application, but the costs-benefits need to be considered for each clinical setting. ${ }^{31}$ Because of the limitation of molecular panel testing worldwide, the BRAF mutation should be used in preference to other mutations in centers with the appropriate laboratory facilities.

Apart from the PCR-based technique, which is the gold standard method for detecting the BRAF mutation, IHC has been introduced as an alternative method. Following the development of the monoclonal antibody, VE1, to direct against the BRAF mutant protein, IHC was used for the analysis of mutations from surgical pathology, with a good outcome of a high sensitivity and specificity reported by some studies. ${ }^{32-34}$ The applications of IHC in the cytology materials from FNAB, or "immunocytochemistry" (ICC), were initially performed by Zimmerman et $\mathrm{al}^{35}$ and Rossi and Schmitt. ${ }^{11}$ 
Zimmerman et a ${ }^{35}$ performed BRAF ICC with the VE1 clone on 55 FNAB cell blocks, which yielded good performance (sensitivity, $93.8 \%$; specificity, $93.8 \%$ ) compared to the ultra-deep sequencing for mutations. Another study by Rossi and Schmitt, ${ }^{11}$ which evaluated BRAF ICC on liquidbased preparations, resulted in an $82 \%$ sensitivity and a $100 \%$ specificity, with a $72 \%$ NPV and a $100 \%$ PPV. Nevertheless, both studies were performed on the malignancy group (Bethesda VI). A recent retrospective analysis by Lee et al, ${ }^{36}$ which included 42 cytologically indeterminate samples from a total of 202 liquid-based preparations, resulted in an $82.3 \%$ sensitivity for the VE1 clone and a $72 \%$ specificity, with a high false-positive rate, for the AUS group, compared with the BRAF mutation from histopathology.

In our study, IHC was also performed to detect the BRAF mutation from the indeterminate category of FNAB by using cell block preparations from the leftover specimen in the needles. With inadequate specimens for the cell block preparations, we could recruit only 20 of the 76 cases. The diagnostic values, demonstrated in Table 3 , show a lower specificity and a higher false positive ( $4 / 20$ cases) than the PCR group.

However, the data were inconclusive due to the small population number available for analysis. The reasons for the relatively high nondiagnostic IHC results in our study may be because an inadequate number of representative tissues were obtained from the FNAB specimens. In the present study, to obtain the cytological material for IHC detection, we performed the FNAB only once. Moreover, those subjects in the cytologically indeterminate group had a smaller proportion of malignant cells than subjects in the malignancy group. We suggest that repeating the needle aspiration and keeping all the materials in the needle, not just the leftover, might give a better yield in the cell block staining.

From our study, we conclude that the detection of the positive BRAF mutation might be useful for making a decision to perform surgery instead of undertaking close follow-up in cases of a cytologically indeterminate category of thyroid FNAB. An additional usefulness is the decision for the extent of surgery (ie, a one-staged total thyroidectomy vs a two-staged surgery). Moreover, the PCR-based method was more effective than the ICC for the detection of the mutation when considering the detailed diagnostic value.

Another application is to use the BRAF mutation as a prognostic factor for thyroid cancer. In a large meta-analysis by Xing et al, ${ }^{7,10}$ the BRAF mutation was associated with higher recurrence and mortality rates of PTC. Another analysis by Liu et $\mathrm{al}^{37}$ found that the BRAF V600E mutation correlated with a lower overall survival rate and aggressive histopathology features and clinical outcomes, including extrathyroidal extension, a higher TNM stage, lymph node metastasis, and a higher recurrence rate.

In our study, the BRAF mutation was detected in 13 of the 76 indeterminate nodules. Twenty-four patients with PTC and one patient with follicular carcinoma were eventually diagnosed from the surgical resection specimens. The mean follow-up time in our study for differentiated thyroid cancer patients was 32 months. Nonetheless, several histopathology and clinical outcomes in these patients were not significantly related to the BRAF mutation. The discordant results obtained from the preoperative FNAB and surgical histopathology may be due to different methods of detection or a distinct mutation rate. Thus, a refinement of the preoperative BRAF mutation method must be developed to ensure that the prognostic value of FNAB has the same yield as a standard surgical specimen.

The reclassification of some thyroid neoplasms as noninvasice follicular variant thyroid neoplasm with papillary-like nuclear features (NIFTP) had an impact on the malignancy rate in each diagnostic category. ${ }^{13}$ In the 2017 Bethesda, ${ }^{15}$ the rate of malignancy depended on the definition of NIFTP as equal, or not equal, to malignancy. In our study, the malignancy rate increased from $16.7 \%$ (NIFTP $\neq$ malignancy) to $20 \%$ (NIFTP $=$ malignancy) in the AUS group. Nonetheless, there was no effect on the malignancy rate in the SUS group even though it tended to be high compared to the risk of malignancy in the 2017 Bethesda. Since NIFTP is most often associated with the indeterminate categories (AUS, FN/SFN, and SUS), ${ }^{38}$ the permanent sections were reviewed using the definitions for diagnosis of NIFTP. Two cases were reclassified as NIFTP and confirmed by negative BRAF staining. In particular, many of the NIFTP cases were noted to have distinct molecular alterations that were related to RAS gene mutations, BRAF K601E mutations, and PPAR $\gamma$ and THADA gene fusions. ${ }^{39-42}$ Hence, the molecular diagnosis might be helpfully applied as another diagnostic criterion for confirmation of the NIFTP diagnosis and be of benefit to management planning.

\section{Limitations}

There were some potential limitations of this study. First, the tissue for the molecular diagnoses may not have accurately represented the true site diagnosed as being an indeterminate nodule. This is because the FNAB was performed twice on the same nodule but on different occasions. In detail, the diagnostic FNAB was done in an outpatient setting, and only the cytologically indeterminate thyroid nodule was included. 
Subsequently, the intraoperative FNAB was performed just before the incision for the BRAF mutation analysis. Second, since ultrasonography was not applied in all FNAB cases, we excluded incidental microcarcinoma detected in other benign diseases. Finally, there was no standardized grading system for the IHC of the FNAB specimens. Moreover, all the results were determined by only one thyroid cytopathological expert who was blinded to the PCR results.

\section{Conclusion}

In recent decades, several molecular panels have been developed to improve the diagnostic value of thyroid cancer. Most tests require sophisticated laboratory work that is not available worldwide. In addition, the costs-benefits of the commercial tests need to be individually assessed. Because of its high accuracy and specificity, the PCR-based BRAF mutation method is a reliable technique for PTC detection, and it could initially be used as the main molecular marker in a tertiary care hospital. Generally, the role of BRAF as a "rule in" test in the AUS is limited due to the low prevalence of cancer in this category. Nevertheless, the fact that the PCR-based BRAF mutation method has a 100\% PPV means that a firm decision can be made to treat the disease as a malignancy. The method can also be used in selected cases, such as in response to a patient's preference or in the presence of high-risk ultrasound features. On the other hand, the BRAF mutation is beneficial in cases of SUS. With positive results and clinical indications, a total thyroidectomy is justified instead of two-staged surgery. The BRAF IHC had limited value in our study due to tissue inadequacy and low specificity. Technical refinement will still be needed for this technique. Our results showed no correlation of the cytological BRAF mutation with several adverse histopathology and clinical outcomes. As to NIFTP, the BRAF mutation was negative, and such a finding can be used as a supplementary criterion for diagnosis.

\section{Acknowledgments}

Our grateful appreciation is expressed to Dr Chulaluck Komoltree, our consulting statistician, and Ms Jeerapa Kerdnoppakhun, our research assistant, for their kind contributions. We also thank all staff of the Department of Otorhinolaryngology, Siriraj Hospital, as well as all patients who were involved in this study. This study was supported in part by a grant from the Faculty of Medicine, Siriraj Hospital, Mahidol University (R0159330028).

\section{Disclosure}

The authors report no conflicts of interest in this work.

\section{References}

1. Mazzaferri EL. Management of a solitary thyroid nodule. N Engl J Med. 1993;328(8):553-559.

2. Agate L, Lorusso L, Elisei R. New and old knowledge on differentiated thyroid cancer epidemiology and risk factors. J Endocrinol Invest. 2012;35(6 Suppl):3-9.

3. Cibas ES, Ali SZ. The Bethesda system for reporting thyroid cytopathology. Thyroid. 2009;19(11):1159-1165.

4. Haugen BR, Alexander EK, Bible KC, et al. 2015 American thyroid association management guidelines for adult patients with thyroid nodules and differentiated thyroid cancer: the American thyroid association guidelines Task Force on thyroid nodules and differentiated thyroid cancer. Thyroid. 2016;26(1):1-133.

5. Zhang M, Lin O. Molecular testing of thyroid nodules: a review of current available tests for fine-needle aspiration specimens. Arch Pathol Lab Med. 2016;140(12):1338-1344.

6. Nikiforova MN, Nikiforov YE. Molecular diagnostics and predictors in thyroid cancer. Thyroid. 2009;19(12):1351-1361.

7. Xing M, Alzahrani AS, Carson KA, et al. Association between BRAF V600E mutation and mortality in patients with papillary thyroid cancer. JAMA. 2013;309(14):1493-1501.

8. Kimura ET, Nikiforova MN, Zhu Z, et al. High prevalence of BRAF mutations in thyroid cancer: genetic evidence for constitutive activation of the RET/PTC-RAS-BRAF signaling pathway in papillary thyroid carcinoma. Cancer Res. 2003;63(7):1454-1457.

9. Xing M, Clark D, Guan H, et al. BRAF mutation testing of thyroid fineneedle aspiration biopsy specimens for preoperative risk stratification in papillary thyroid cancer. J Clin Oncol. 2009;27(18):2977-2982.

10. Xing M, Alzahrani AS, Carson KA, et al. Association between BRAF V600E mutation and recurrence of papillary thyroid cancer. $J$ Clin Oncol. 2015;33(1):42-50.

11. Rossi ED, Schmitt F. Pre-analytic steps for molecular testing on thyroid fine-needle aspirations: the goal of good results. Cytojournal. 2013; 10:24.

12. Dvorak K, Aggeler B, Palting J, et al. Immunohistochemistry with the anti-BRAF V600E (VE1) antibody: impact of pre-analytical conditions and concordance with DNA sequencing in colorectal and papillary thyroid carcinoma. Pathology. 2014;46(6):509-517.

13. Nikiforov YE, Seethala RR, Tallini G, et al. Nomenclature revision for encapsulated follicular variant of papillary thyroid carcinoma: a paradigm shift to reduce overtreatment of indolent tumors. JAMA Oncol. 2016;2(8):1023-1029.

14. Seethala RR, Baloch ZW, Barletta JA, et al. Noninvasive follicular thyroid neoplasm with papillary-like nuclear features: a review for pathologists. Mod Pathol. 2018;31(1):39-55.

15. Cibas ES, Ali SZ, The ASZ. The 2017 Bethesda system for reporting thyroid cytopathology. Thyroid. 2017;27(11):1341-1346.

16. Yanus GA, Belyaeva AV, Ivantsov AO, et al. Pattern of clinically relevant mutations in consecutive series of Russian colorectal cancer patients. Med Oncol. 2013;30(3):686.

17. Chat-Uthai N, Vejvisithsakul P, Udommethaporn S, et al. Development of ultra-short PCR assay to reveal BRAF V600 mutation status in Thai colorectal cancer tissues. PLoS One. 2018;13(6):e0198795.

18. Wiltshire JJ, Drake TM, Uttley L, Balasubramanian SP. Systematic review of trends in the incidence rates of thyroid cancer. Thyroid. 2016;26(11):1541-1552.

19. Pathak KA, Leslie WD, Klonisch TC, Nason RW. The changing face of thyroid cancer in a population-based cohort. Cancer Med. 2013; 2(4):537-544.

20. Su X, Jiang X, Xu X, et al. Diagnostic value of BRAF (V600E)-mutation analysis in fine-needle aspiration of thyroid nodules: a meta-analysis. Onco Targets Ther. 2016;9:2495-2509.

21. Xing M, Haugen BR, Schlumberger M. Progress in molecular-based management of differentiated thyroid cancer. Lancet. 2013;381(9871): 1058-1069.

22. Cohen $Y$, Xing M, Mambo E, et al. BRAF mutation in papillary thyroid carcinoma. J Natl Cancer Inst. 2003;95(8):625-627. 
23. Nikiforov YE, Nikiforova MN. Molecular genetics and diagnosis of thyroid cancer. Nat Rev Endocrinol. 2011;7(10):569-580.

24. Xing M. BRAF mutation in thyroid cancer. Endocr Relat Cancer. 2005; 12(2):245-262.

25. Chung K-Wook, Yang SK, Lee GK, et al. Detection of BRAF ${ }^{\mathrm{V} 600 \mathrm{E}}$ mutation on fine needle aspiration specimens of thyroid nodule refines cyto-pathology diagnosis, especially in $\mathrm{BRAF}^{\mathrm{V} 600 \mathrm{E}}$ mutation-prevalent area. Clin Endocrinol. 2006;65(5):660-666.

26. Khemkha A. Correlation between BRAF mutation and clinicopathological features of papillary thyroid cancer in Thai patients [dissertation]. Bangkok: Chulalongkorn University; 2008.

27. Guan H, Ji M, Bao R, et al. Association of high iodine intake with the T1799A BRAF mutation in papillary thyroid cancer. JClin Endocrinol Metab. 2009;94(5):1612-1617.

28. Xing M. Molecular pathogenesis and mechanisms of thyroid cancer. Nat Rev Cancer. 2013;13(3):184-199.

29. Jinih M, Foley N, Osho O, et al. BRAF ${ }^{\mathrm{V} 600 \mathrm{E}}$ mutation as a predictor of thyroid malignancy in indeterminate nodules: A systematic review and meta-analysis. Eur J Surg Oncol. 2017;43(7):1219-1227.

30. Nikiforov YE, Steward DL, Robinson-Smith TM, et al. Molecular testing for mutations in improving the fine-needle aspiration diagnosis of thyroid nodules. J Clin Endocrinol Metab. 2009;94(6):2092-2098.

31. Nikiforov YE, Ohori NP, Hodak SP, et al. Impact of mutational testing on the diagnosis and management of patients with cytologically indeterminate thyroid nodules: a prospective analysis of 1056 FNA samples. J Clin Endocrinol Metab. 2011;96(11):3390-3397.

32. Capper D, Preusser M, Habel A, et al. Assessment of BRAF V600E mutation status by immunohistochemistry with a mutation-specific monoclonal antibody. Acta Neuropathol. 2011;122(1):11-19.

33. Koperek O, Kornauth $\mathrm{C}$, Capper D, et al. Immunohistochemical detection of the BRAF V600E-mutated protein in papillary thyroid carcinoma. Am J Surg Pathol. 2012;36(6):844-850.
34. Ghossein RA, Katabi N, Fagin JA. Immunohistochemical detection of mutated BRAF V600E supports the clonal origin of BRAF-induced thyroid cancers along the spectrum of disease progression. $J$ Clin Endocrinol Metab. 2013;98(8):E1414-E1421.

35. Zimmermann AK, Camenisch U, Rechsteiner MP, Bode-Lesniewska B, Rössle M. Value of immunohistochemistry in the detection of $\mathrm{BRAF}(\mathrm{V} 600 \mathrm{E})$ mutations in fine-needle aspiration biopsies of papillary thyroid carcinoma. Cancer Cytopathol. 2014;122(1):48-58.

36. Lee SR, Yim H, Han JH, et al. VE1 antibody is not highly specific for the BRAF V600E mutation in thyroid cytology categories with the exception of malignant cases. Am J Clin Pathol. 2015;143(3):437-444.

37. Liu C, Chen T, Liu Z. Associations between BRAF(V600E) and prognostic factors and poor outcomes in papillary thyroid carcinoma: a meta-analysis. World J Surg Oncol. 2016;14(1):241.

38. Faquin WC, Wong LQ, Afrogheh AH, et al. Impact of reclassifying noninvasive follicular variant of papillary thyroid carcinoma on the risk of malignancy in the Bethesda system for reporting thyroid cytopathology. Cancer Cytopathol. 2016;124(3):181-187.

39. Rivera M, Ricarte-Filho J, Knauf J, et al. Molecular genotyping of papillary thyroid carcinoma follicular variant according to its histological subtypes (encapsulated vs infiltrative) reveals distinct BRAF and ras mutation patterns. Mod Pathol. 2010;23(9):1191-1200.

40. Afkhami M, Karunamurthy A, Chiosea S, et al. Histopathologic and Clinical Characterization of Thyroid Tumors Carrying the BRAF(K601E) Mutation. Thyroid. 2016;26(2):242-247.

41. Kim TH, Lee M, Kwon AY, et al. Molecular genotyping of the noninvasive encapsulated follicular variant of papillary thyroid carcinoma. Histopathology. 2018;72(4):648-661.

42. Hung YP, Barletta JA. A user's guide to non-invasive follicular thyroid neoplasm with papillary-like nuclear Features (NIFTP). Histopathology. 2018;72(1):53-69.
OncoTargets and Therapy

\section{Publish your work in this journal}

OncoTargets and Therapy is an international, peer-reviewed, open access journal focusing on the pathological basis of all cancers, potential targets for therapy and treatment protocols employed to improve the management of cancer patients. The journal also focuses on the impact of management programs and new therapeutic agents and protocols on

\section{Dovepress}

patient perspectives such as quality of life, adherence and satisfaction. The manuscript management system is completely online and includes a very quick and fair peer-review system, which is all easy to use. Visit http://www.dovepress.com/testimonials.php to read real quotes from published authors. 